\title{
AUTOMATIC IDENTIFICATION AND CLASSIFICATION OF MICROANEURYSMS FOR DETECTION OF DIABETIC RETINOPATHY
}

\author{
Gowthaman $\mathbf{R}^{1}$ \\ ${ }^{1}$ Coimbatore, Tamilnadu, India
}

\begin{abstract}
Diabetic Retinopathy is major cause for visual loss and visual impaired vision worldwide. A proper detection and treatment of this disease in needed in time. Microaneurysms detection is difficult process because they appeared as a first sign of diabetic retinopathy disease. In past few years, many approaches raised for the identification and detection of this diseases using some features extraction techniques, mathematical algorithms and artificial neural network classifiers which lacks in some drawbacks in preprocessing, extraction of appropriate features, blood vessels extraction and in chosing classification techniques. This paper is developed to prefectly detect the candidate regions by using Gabor filter bank and separation of blood vessels from the retina image. Then for each candidate region different feature vectors are extracted. These features are given to multi class classifier for training and testing. Performance of this proposed work is evaluated with performance metrics such as accuracy, sensitivity, specificity and execution time and proved as a successful method for automatic early detection of diabetic retinopathy.
\end{abstract}

Keywords: Microaneurysms (MAs), Diabetic Retinopathy (DR), Support Vector Machine (SVM), Extreme Learning Machine (ELM).

\section{INTRODUCTION}

Eye is a very essential and critical organ of the human body which only gives vision. It is a complex organ next to human brain. There are huge eye diseases spreading nowadays due to improper care. Among those diseases Diabetic Retinopathy (DR) is severe and wide spreading diseases. It has been identified as one of the cause for blindness or vision impairment.

According to recent estimates, approximately 285 million people worldwide $(6.6 \%)$ in the $20-79$ year age group have diabetes in 2010 and by 2030, 438 million people (7.8\%) of the adult population, is expected to have diabetes [1]. And one noticeable thing is India at first position of 50.8 million people affected by diabetics by the survey taken on 2010 [2].

Thus there is a much urged need to control and early detection of this disease. Early detection and management of risk factors responsible for diabetic retinopathy could postpone development of diabetic retinopathy or control its progression.

Microaneurysms (MAs) are among the earliest clinical signs of diabetic retinopathy [4], and [5]. They arise due to high sugar levels in the blood. MAs are of small, almost round and red in color.The next sign of DR is hemorrhages which are also referred to as dot or blot hemorrhages. When the wall of thin vessels or MAs is sufficiently weakened, it may rupture and give rise to a hemorrhages. Dot hemorrhages appear as bright small red dots and blot hemorrhages are larger red
lesions.Sometimes dot hemorrhages and Mas are considered as a single red lesion class known as HMAs [3].

The initial detection of this disease can be done manually. But it is very tough and waste of time and not sure about the accurate detection. This urged to develop automated techniques which are probably accurate and more number of images can be processed together. For this huge techniques are proposed by many authors for early detection of DR in Image Processing.

The retinal image of the patients affected by this disease is captured intially. Those images are subjected first for pre processing because they may be in low resolution and noisy. Then from pre processed image the features are extracted for the identification. Feature extraction is an important technique in which appropriate and effective features must be extracted which only helps for perfect identification. From those features they are finally given for the identification and classification technique.

This Computer aided diagnostic systems for eye diseases use digital retina images which are an essential mean to document and diagnose various eye diseases in clinics.Such a system should be able to distinguish between affected retina images and normal retina images.This will significantly reduce the work load for the ophthalmologists as they have to examine only those images diagnosed by the system as potential anomalies containing affected retina [3]. 
The paper is organized as follows: section 2 gives the related works; section 3 explains detailed about the proposed work which involves pre processing of the retina image; feature extraction, identification and classification; section 4 presents the experimental results and finally section 5 provides conclusion and future extension of this work.

\section{RELATED WORK}

Automated microaneurysm detection was first attempted in the early 1980s, and was on fluorescein angiographic images. Lay et al [11] illustrated a morphological approach to MA detection. Image resolution and dynamic range were low $(256 \times 256$ pixels with 100 gray levels); Eventhough, by means of imaging microscope to digitize film negatives, the digital image covered a small field-of-view of the macula so that MAs were well resolved. As negatives were used, the MAs appeared as dark dots, thus as localized minima in the intensity image. A top hat transform was applied to identify the local minima. The automated MA detector achieved nearly $60 \%$ sensitivity with 4.2 false detections per image for detecting and locating MAs.

Author Hipwell et al [12] modified past microaneurysm detector system. They achieved $43 \%$ sensitivity at 0.11 false positives per image, the lower sensitivity reflecting the greater difficulty to visualise microaneurysms without fluorescein angiography. Using the presence of one or more microaneurysms in an image to indicate the presence of diabetic retinopathy their system achieved a disease detection sensitivity of $85 \%$ with a specificity of $76 \%$. They demonstrated in a controlled clinical trial that their method could be useful in a screening situation to identify $50 \%$ of normal retina.

Low-resolution colour retinal images was investigated by Yang et al [13], but removed the matched-filtering stage and replaced it with an extra dilation applied after the opening image reconstruction (within the top hat transform) using a $3 \times 3$ structuring element. They achieved a microaneurysm detection sensitivity of $85 \%$ with a specificity of $90 \%$ but used a testing procedure that is quite disputable. They also report a preliminary sensitivity of $90 \%$ to detect at least one true microaneurysm in a training set of 46 normal and diseased images of various qualities

Fleming et al [14] proposed a local contrast normalization based method. They used watershed transform to detect MAs by distinguishing between MAs and other dots present on retina. The reported sensitivity and specificity were $85.4 \%$ and $83.1 \%$, respectively. An online competition for MAs detection with the name of Retinopath Online Challenge (ROC) is introduced by the University of Iowa and ROC organizers [15]. The purpose is to improve the quality of computer aided and automated diagnoses of DR.The results of first international competition were reported in [16].

\section{PROPOSED WORK}

Many methods are followed for the detection of diabetic retinopathy. And some methods followed by identification and classification of MAs for detection of diabetic retinopathy. Here this proposed work also follows by detection of MAs for identification of diabetic retinopathy. This work is composed of mainly three main processes of preprocessing, feature extraction and classification.

The block diagram of this work is shown in figure 1 which gives complete details of this whole process. The preprocessing phase removes noises, enhances the image and extracts candidate regions for MAs. Then the feature extraction phase extracts features in different properties for each candidate lesions and followed by classification phase which identifies MAs and Non MAs separately. To prove this works efficiency results are displayed finally.

\subsection{Pre Processing}

Pre processing is an initial and vital process in any image processing based system. This process makes the image prepared for the further extraction of features and for classification.

Here, the captured retina images are at low light and there is maximum chance of noise to affect images. In this pre processing the images are denoised and enhanced.

At first they are given for noise removal technique. In medical image processing noise removal is crucial step because this may give the chance to affect the result widely. There are various noise removal techniques available in image processing. 


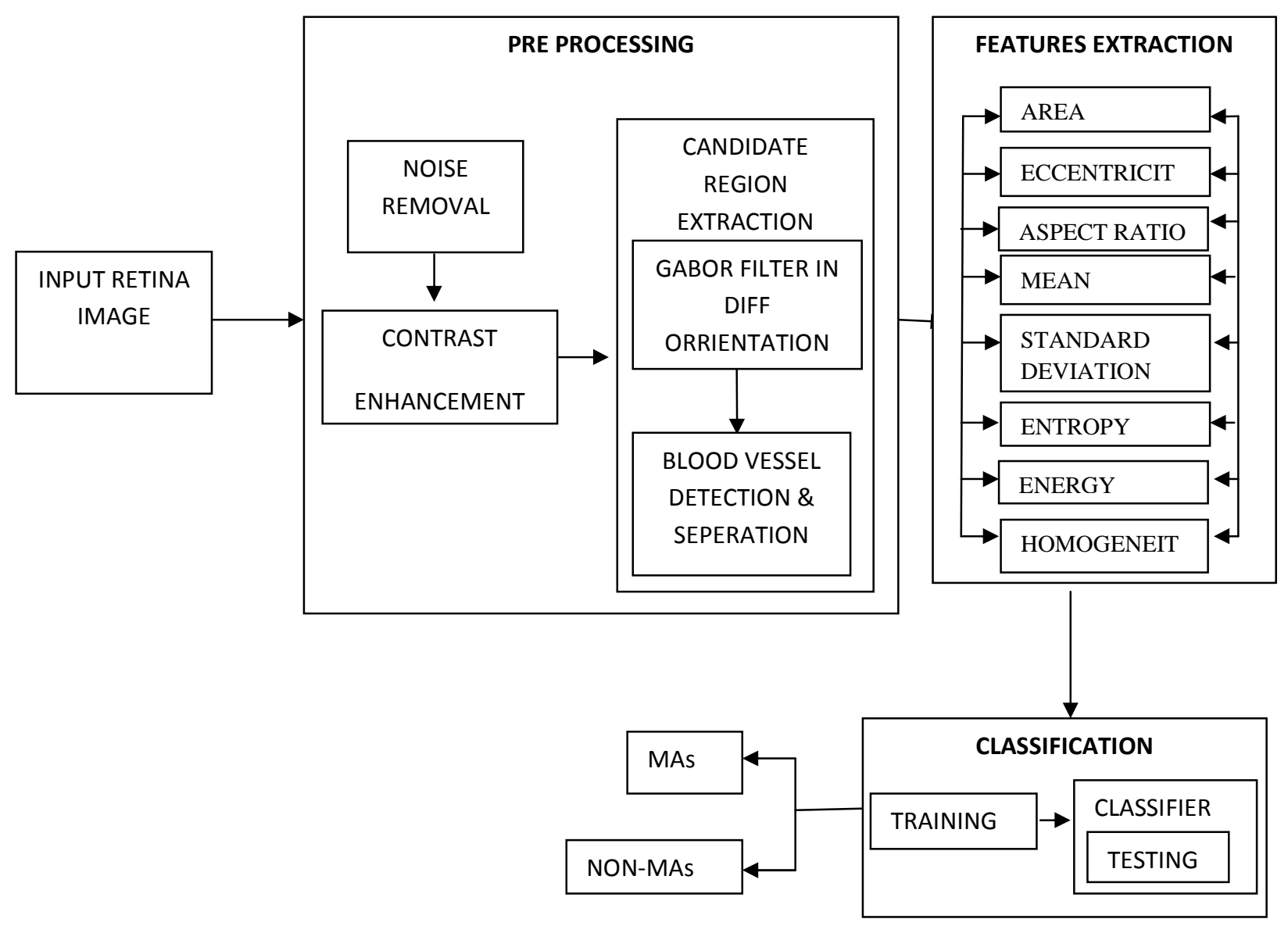

Fig-1: Block Diagram of the Proposed Work

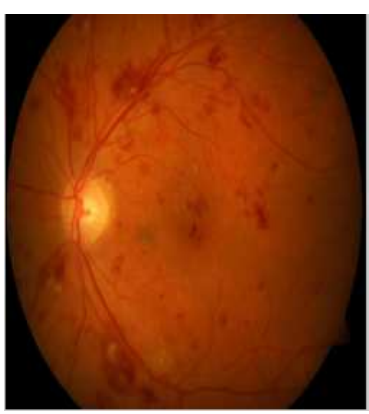

Fig 2.1: Original Input Retinal Image

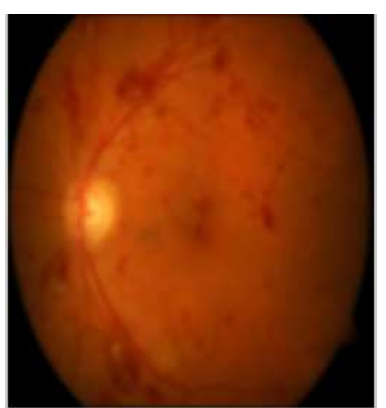

Fig 2.2: Smothing by Morpological Operation 


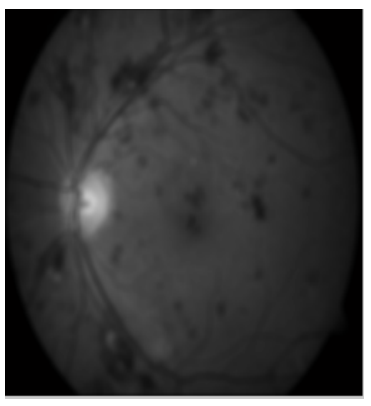

Fig 2.3: Obtained Green Channel Image

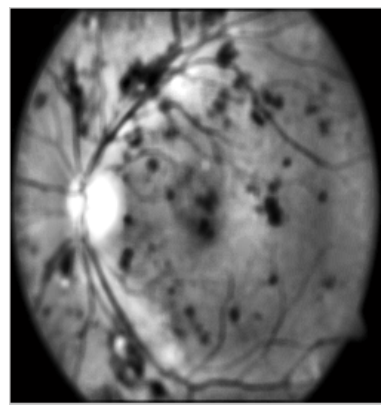

Fig 2.4: Contrast Enhanced Image

Fig -2: Preprocessing Output

Here Gaussian filter is chosen among them because it is efficient in removal of noise and for smoothing the image. Then after removal of noise the morphological operations of opening and closing are done with the Structuring Element (SE) which is defined in equation (3.1 and 3.2).

$$
\begin{aligned}
& \text { Opening: } \gamma^{(s B)}(f)=\delta^{(s B)}\left[\epsilon^{(s B)}(f)\right] \\
& \text { Closing: } \emptyset^{(s B)}(f)=c^{(s B)}\left[\delta^{(s B)}(f)\right]
\end{aligned}
$$

Where $\mathrm{sB}$ is taken as structuring element $\mathrm{B}$ of size $\mathrm{s}$. $\mathrm{f}$ is the gray level image. This gives smooth retina region which contains dark red lesions and Optical Vessels. But the lesions are not improved to maximum contrast.

MAs are dark red dot they are highly visual in green plane. Thus from the smoothed image only green channel image is extracted from the RGB Image which used for further processing.

To improve the lesions for easy detection adaptive contrast enhancement transformation is done.This is performed by $\mathrm{w} \times \mathrm{w}$ sliding window with assumption that $\mathrm{w}$ is large enough to contain a statistically representative distribution of the local variation of lesions [6].

$$
g=255 \frac{\left[\Psi_{\mathrm{W}}(\mathrm{f})-\Psi_{\mathrm{W}}\left(\mathrm{f}_{\min }\right)\right]}{\left[\Psi_{W}\left(f_{\max }\right)-\Psi_{W}\left(f_{\min }\right)\right]}
$$

Where the sigmoidal function for a window defined as

$$
\Psi_{W}(f)=\left[1+\exp \left(\frac{m_{W}-f}{\sigma_{W}}\right)\right]^{-1}
$$

While $f_{\text {max }}$ and $f_{\text {min }}$ are the maximum and minimum intensity of the smooth green channel image, respectively. $\mathrm{m}_{\mathrm{W}}$ and $\sigma_{\mathrm{W}}$ is the mean and variance of intensity values within the window.

\subsubsection{Candidate Region Extraction}

Candidate region (lesion) is a small circular object which is dark red dot and patchs in retinal image. By our naked eye it can be able to identify but they varies based on its texture, contrast and blood vessels in the image makes difficult to identify it clearly. In this phase they are extracted by gabor filter and blood vessels are segmented to extract it without any difficulty.

\subsubsection{Gabor Filter}

The contrast enhanced image is given for Gabor filter banks for enhancement of lesions.Gabor filters are Famous due to their fine frequency tuning and orientations electiveness. They are appropriate for texture representation and discrimination [7]. Gabor filter is represented by a Gaussian kernel function which can model a wide range of shapes depending upon the values of its parameters [7] which is shown in equation (3.5). This property makes them suitable for MAs and dot hemorrhage detection.

$$
\begin{aligned}
G(x, y, \sigma, \Omega, \theta, r)= & \frac{1}{\sqrt{\pi r \sigma}} e^{-\left(\frac{1}{2}\right)\left[\left(\frac{d_{1}}{\sigma}\right)^{2}+\left(\frac{d_{2}}{\sigma}\right)^{2} \mathrm{I}\right.}\left(d_{1}(\cos \Omega\right. \\
& +l \sin \Omega))
\end{aligned}
$$

Where $\sigma, \Omega$ and $r$ are the standard deviations of gaussian, spatial frequency and aspect ratio respectively, $y$ is the orientation of filter and $\mathrm{d}_{1}=\mathrm{x} \cos \theta+\mathrm{y} \sin \theta$ and $\mathrm{d}_{2}=$ $-\mathrm{x} \sin \theta+\mathrm{y} \cos \theta$. The contrast enhanced image $\mathrm{g}$ is convolved with Gabor filter $\mathrm{G}$ centered at location(s, $\mathrm{t}$ ) to generate Gabor filter response $\mathrm{g}$ for selected values of $\mathrm{s}, \mathrm{O}$ and $\mathrm{y}$ is given in the following equation (3.6) [7]. 


$$
\gamma(\sigma, \Omega, \theta)=\sum_{x} \sum_{y} g(x, y) G(s-x, t-y, \sigma, \Omega, \theta, r)
$$

To obtaine maximum scale and frequecy values, the maximum Gabor filter bank response $\mathrm{M}_{\gamma}(\sigma, \Omega)$ is computed by the equation for $\theta$ spanning $45^{\circ}, 90^{\circ}, 135^{\circ}$ and $180^{\circ}$ defined in equation (3.7).

$$
M_{\gamma}(\sigma, \Omega)=\max |\gamma(\sigma, \Omega, \theta)|
$$

From this binary candidate regions for MAs and $\mathrm{H}$ are extracted by applying a small threshold value $\mathrm{T}_{1}$. These regions contain actual lesion region, false lesion regions and blood vessels also. From this blood vessels and false lesion must be removed before given for further process.

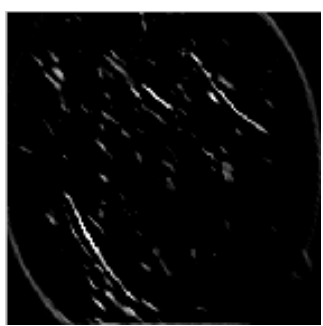

Fig -3.1: Orientation at 45 degree

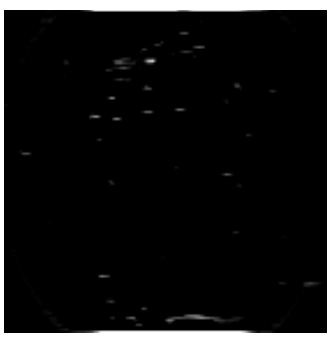

Fig -3.3: Orientation at 135 degree

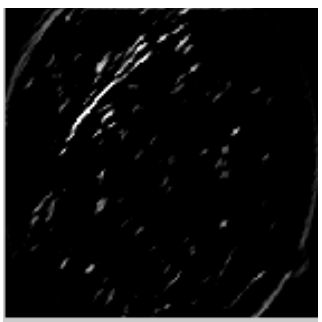

Fig -3.2: Orientation at 90 degree

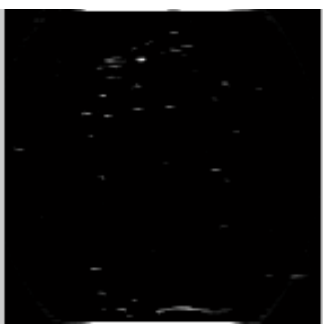

Fig -3.4: Orientation at 180 degree
Fig -3: Gabor Filter Response at Different Orientations

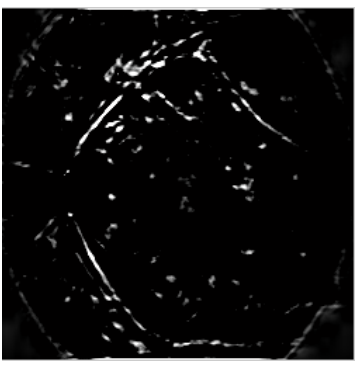

Fig -4: Segmented Red Lesions Containing Spurious Region

\subsubsection{Detection and Removal of Blood Vessels}

Blood vessels are important structure in retinal images. It contains enough information for the localization of some anchor points and it maps the whole retina.

For the diagnosis or evaluation of ocular or systemic diseases, examination of retinal blood vessels is important. It offers much information however for easy detection of pathological lesions like exudate or microaneurysms it must be excluded.

Blood vessel segmentation is crucial process they are segmented basically based on mainly three approaches: thresholding method, tracking method and machine trained classifier [8]. Here threshold based blood vessel segmentation is followed.

It composed of four steps, matched filtering, entropy based thresholding, length filtering, and vascular intersection detection proposed in [9].

\subsubsection{Matched Filtering}

To detect the piecewise linear segments of blood vessels in retinal images, matched filtering is performed. It is designed in two dimensions for the retinal images which enhance the blood vessels. A prototype matched filter is expressed as in equation (3.8).

$$
f(x, y)=-\exp \left(\frac{-x^{2}}{2 \sigma^{2}}\right), \text { for }|y| \leq \frac{L}{2}
$$

Where $\mathrm{L}$ is taken as length of the segment for which the vessel is assumed to have a fixed orientation. Here the direction of the vessel is supposed to be aligned along the y-axis. Because a vessel may be oriented at any angles, the kernel needs to be rotated for all possible angles. A set of twelve $16 \times 15$ pixel kernels is applied by convolving to a retina image and at each pixel only the maximum of their responses is retained.

\subsubsection{Local Entroy Thresholding}

Here the blood vessels segments are extracted from the retinal image. To extract vessels a local entropy based thresholding 
technique is implemented here which helps in preserving the spatial structures in the thresholded/binarized image [10].

The co-occurrence matric of the image $F$ is an $P \times Q$ dimensional matrix $\mathrm{T}=\left[\mathrm{t}_{\mathrm{ij}}\right] \mathrm{P} \times \mathrm{Q}$ which gives an initiative about the transition of intensities between adjacent pixels, indicating spatial structural information of an image. Depending upon the ways in which the gray level i follows gray level $j$, different definitions of cooccurrence matrix are possible. Here, we made the co-occurrence matrix asymmetric by considering the horizontally right and vertically lower transitions.
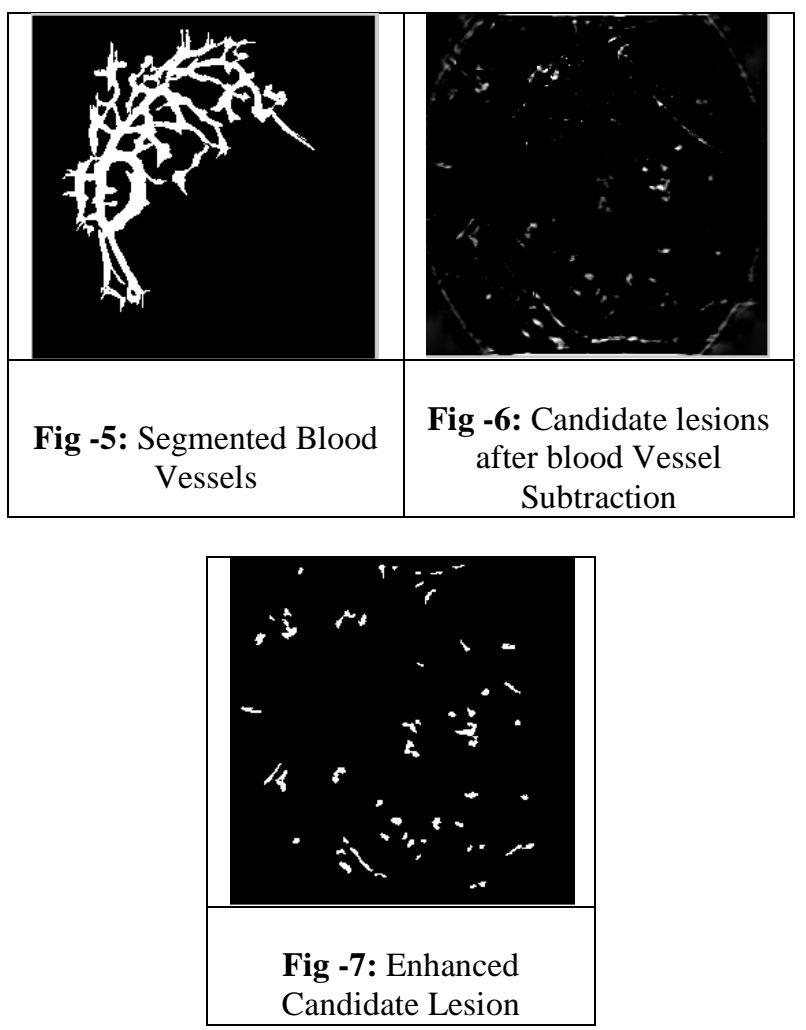

By performing the above four operations bloods vessels are accurately detected and blood vascular patterns are segmented from Gabor filtered image.

\subsection{Feature Vector Construction}

Features are essential for any classification or analysis in image processing. There are several types of features which can be extracted from the images each gives its identical informations about the image. Here MAs region supposed with properties such as shape, color and size which appears as a dark red colored circle shape. To identify MA and non-MA region feature vectors are formed for each candidate regions.

Each Candidate region in the image has their identical feature values which can be taken as potential MA region. If the image $\mathrm{f}$ contains $\mathrm{N}$ number of candidate regions, then $\mathrm{f}=$ $\left\{\mathrm{f}_{1}, \mathrm{f}_{2}, \ldots, \mathrm{f}_{\mathrm{N}}\right\}$. Therefore for the each candidate region contains $\mathrm{k}$ features $\left(\mathrm{f}_{\mathrm{i}}=\left\{\mathrm{k}_{1}, \mathrm{k}_{2}, \ldots ., \mathrm{k}_{\mathrm{N}}\right\}\right.$, where $\left.\mathrm{i}=1,2,3 \ldots \mathrm{N}\right)$.

- Area is the total number of pixels in candidate region.

- Eccentricity is the ratio of the distance between foci of ellipse and its major axis length and it is equal to 0 for a circular region.

- Aspect Ratio is the ratio of major axis length to minor axis length of the candidate region.

- Mean and Standard Deviation value of all green channel pixels within the candidate region.

- Entropy value of all pixels in the square region including candidate region pixels and its neighboring pixels.

- Energy value of all pixels in the square region including candidate region pixels and its neighboring pixels.

- Homogeneity value of all pixels in the square region including candidate region pixels and its neighboring pixels.

\subsection{Classification}

The classification is the final process which classifies the result (I.e, Normal, Abnormal etc). There are various classifiers used in literature which divides into two classes majorly called as dichotomies and some classifies into multi classes (e.g. decision trees [17], feedforward neural networks). Here SVM and ELM classifier is taken to compare the effeciency of proposed work.

\subsubsection{SVM Classification}

Support Vector Machine (SVM) is a useful method for classification of high dimensional problems which suits for only 2 class classification. For multi class classification (K) the classifier has to be trained typically placed in parallel and each one of them is trained to separate one class from the $\mathrm{K}-1$ others. This way of decomposing a general classification problem into dichotomies is known as a one-per-class decomposition, and is independent of the learning method used to train the classifiers. This process is little difficult and lacks in time consumption.

Thus a multi class classification of SVM is chosen here for the classification from Cody Neuburger [18]. In traditional SVM; the structure of trained SVM is formed in a $1 \times 1$ structure. And from that structure the SVM classifier classifies into two classes of 1's and 0's. The user can take on their own choose of 1's as normal or abnormal vise versa based on the training given.

Here in this multi class SVM classification, the candidate region features which are extracted for the dataset previously are divided into two segments for training segment and testing segment. 
The number of training segment $(\mathrm{t})$ and its features (trset) are given for the svmtrain MATLAB function.

$$
\text { svmStruct }=\sum_{1}^{\mathrm{t}} \text { svmtrain(trset, bgroup) }
$$

Where, bgroup is the binarized group in which 1 is the current class and 0 is all other classes. This svmStruct contains $t$ number of structures. For the classification of testing segments, the svmStruct is utilized with testing segments features in svmclassify MATLAB function same as the above equation. It is the Strong classification performance with simple implementation.

\subsubsection{ELM Classification:}

A new learning algorithm for Single Hidden Layer FeedForward Networks (SLFNs), called Extreme Learning Machine (ELM), has been proposed by Huang et al. [21, 22], which helps in solving regression and classification problems. It can also used to reach good solutions analytically, and its learning speed is extremely faster than other traditional methods.

Here, the input weights and biases are determined randomly and they are not updated during training iterations [22]. The activation function like sine, gaussian, sigmoidal etc., can be chosen for hidden neuron layer and linear activation functions for the output neurons. It is a Multi-class classification where number of output neurons will be automatically set equal to number of classes. (For example, if there are 7 classes in all, there will have 7 output neurons; neuron 5 has the highest output means input belongs to 5-th class).

The output weights are obtained by using norm Least Squares (LS) and pseudo inverse of a linear system.

For a given $\mathrm{N}$ arbitrary input-output relation $\left(\mathrm{x}_{\mathrm{i}}, \mathrm{t}_{\mathrm{i}}\right)$, where $x_{i}=\left[x_{i 1}, x_{j 2}, \ldots, x_{i n}\right]^{T} \in R^{n}$ and $t_{i}=\left[t_{i 1}, t_{j 2}, \ldots, t_{i n}\right]^{T} \in R^{m}$, a single layered network with $\widetilde{\mathrm{N}}$ neurons in hidden layer and a given activation function $\mathrm{g}(\mathrm{x})$ is modeled as

$$
\sum_{i=1}^{\widetilde{N}} \beta_{i} g\left(w_{i} \cdot x_{j}+b_{i}\right)=o_{j}, j=1, \ldots, N
$$

Where $\mathrm{w}_{\mathrm{i}}=\left[\omega_{\mathrm{i} 1}, \omega_{\mathrm{i} 2}, \ldots, \omega_{\mathrm{in}}\right]^{\mathrm{T}}$ shows the weight of the $\mathrm{i}^{\text {th }}$ hidden neuron, and $\beta_{\mathrm{i}}=\left[\beta_{\mathrm{i} 1}, \beta_{\mathrm{i} 2}, \ldots, \beta_{\mathrm{in}}\right]^{\mathrm{T}}$ shows the weight of the $\mathrm{i}^{\text {th }}$ hidden neuron to the output neurons, and $b_{i}$ is the bias of the $\mathrm{i}^{\text {th }}$ hidden neuron.

The SLFNs which is defined in Eq. (3.10) can be approximated with zero error [22]. In other words, the Eq. (3.10) can be rewritten in the following form:

$$
\mathrm{H} \beta=\mathrm{T}
$$

The description of equation (3.11) is explained in [23]. After calculation of $\mathrm{H}$, the output weights are determined by using equation (3.12).

$$
\beta=H^{*} \times T
$$

\section{EXPERIMENTAL RESULTS}

To evaluate the proposed work for the identification of diabetic retinopathy using detection of MAs is performed using the retina images taken from publicly available databases such as DRIVE [19], and DIARETDB1 [20] in MATLAB environment. The results obtained from the SVM and ELM classification is compared and discussed here.

Table -1: Dataset Specification

\begin{tabular}{|l|l|l|l|}
\hline Dataset & $\begin{array}{l}\text { Total } \\
\text { Images }\end{array}$ & $\begin{array}{l}\text { Training } \\
\text { Segment }\end{array}$ & $\begin{array}{l}\text { Testing } \\
\text { Segment }\end{array}$ \\
\hline DRIVE & 40 & 20 & 20 \\
\hline DIARETDB1 & 89 & 45 & 44 \\
\hline
\end{tabular}

The datasets specified in table 1 is taken for the evaluation because these dataset images contain varieties of DR lesions which suits well for evaluation. The results are taken from both the dataset which contains both normal and abnormal (i.e contains lesions) images that are equally divided for both training and testing.

Initially the training set features are given to the svm classifier which produces the svm structure that is saved separately. Then testing set is given to the system which follows same procedure as training set till feature extraction. Then in classification, the structured svm which is saved before is loaded and based on this structure, the classifier classifies the testing images individually. The svm classifier produces the result based on its feature values that match approximately with the feature values from the trained images. (For example, we take one single test image features, if its features match in svm classifier with the features of 5 in the trained image, then the result will be 5).

As SVM classification, in ELM training along with its Number of Hidden Neurons, Activation Function, Type- 0 for regression; 1 for (both binary and multi-classes) classification is given as input for the ELM training. It produces output of ELM model.

This ELM model is given for the testing along with the testing features of MAs to the classifier. That model helps to predict correctly the testing image features candidate lesions as MAs, Non-MAs accurately. 


\subsection{Performance Metrics}

For the evaluation of this proposed work the performance metrics such as Sensitivity (Sen), Specificity (Spec), Positive Predictive Value (PPV) and Accuracy (Acc) is taken.These parameters are calculated using the following equations respectively:

$$
\begin{aligned}
& \operatorname{Sen}(\%)=\frac{\mathrm{TP}}{(\mathrm{TP}+\mathrm{FN})} \times 100 \\
& \operatorname{Spec}(\%)=\frac{\mathrm{TN}}{(\mathrm{TN}+\mathrm{FP})} \times 100 \\
& \operatorname{PPV}(\%)=\frac{\mathrm{TP}}{(\mathrm{TP}+\mathrm{FP})} \times 100 \\
& \operatorname{Acc}(\%)=\frac{(\mathrm{TP}+\mathrm{TN})}{(\mathrm{TP}+\mathrm{TN}+\mathrm{FP}+\mathrm{FN})} \times 100
\end{aligned}
$$

Where

- TruePositive (TP): MA regions that are correctly classified by the classifier.

- FalsePositive (FP): Non-MA regions that are wrongly classified as MA regions by the classifier.

- TrueNegative (TN): Non-MA regions that are correctly classified bythe classifier.

- FalseNegative (FN): MA regions that are wrongly classified as non-MA regions by the classifier.

Table -2: Performance assessment between SVM and ELM for MAs detection for DRIVE

\begin{tabular}{|l|l|l|}
\hline Factors & SVM & ELM \\
\hline TP & 90 & 92 \\
\hline TN & 140 & 143 \\
\hline FP & 6 & 3 \\
\hline FN & 4 & 2 \\
\hline Sen $(\%)$ & 95.74 & 97.87 \\
\hline Spec $(\%)$ & 95.89 & 97.94 \\
\hline PPV (\%) & 93.75 & 96.84 \\
\hline Acc (\%) & 95.83 & 97.91 \\
\hline
\end{tabular}

The results presented in the table 2 compares the performance of the classifier SVM and ELM for the detection of MAs. It clearly reveals that proposed technique with ELM classifier outperforms when compared with SVM in Sensitivity, Specificity, PPV and Accuracy for DRIVE dataset.

Table -3: Performance assessment between SVM and ELM for MAs detection for DIARETDB1

\begin{tabular}{|l|l|l|}
\hline Factors & SVM & ELM \\
\hline TP & 154 & 159 \\
\hline TN & 230 & 237 \\
\hline
\end{tabular}

\begin{tabular}{|l|l|l|}
\hline FP & 11 & 4 \\
\hline FN & 15 & 10 \\
\hline Sen $(\%)$ & 91.12 & 94.08 \\
\hline Spec $(\%)$ & 95.43 & 98.34 \\
\hline PPV (\%) & 93.33 & 97.54 \\
\hline Acc (\%) & 93.65 & 96.58 \\
\hline
\end{tabular}

Here from the table 3, the performance of the classifier SVM and ELM for the detection of MAs is compared. It clearly highlights that proposed technique with ELM classifier outperforms when compared with SVM in Sensitivity, Specificity, PPV and Accuracy for DIARETDB1 dataset.

The execution time is also another factor which must be minimum because any proposed system must satisfy in the means of both efficiency and time.

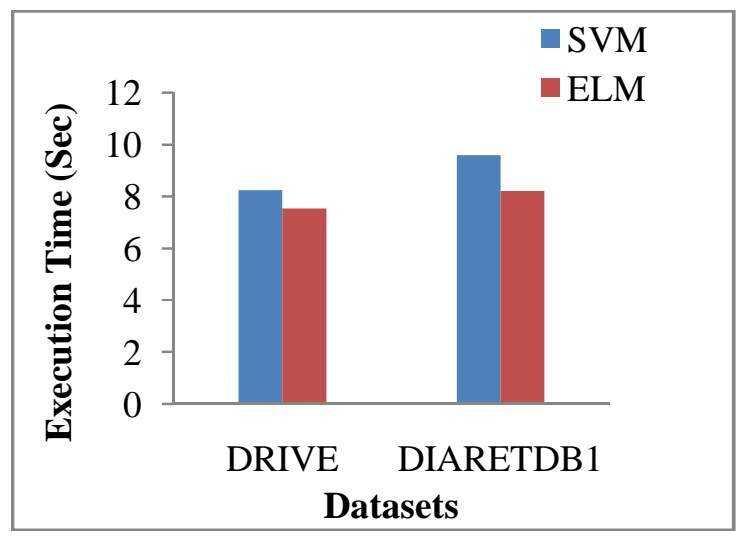

Chart-1: Comparison of Execution Time for datasets

The above figure shows the comparison of time taken by the proposed work to complete its process for the dataset DRIVE and DIARETDB1. From the chart ELM with the proposed work archives efficient result in reduced time than SVM for the both dataset.

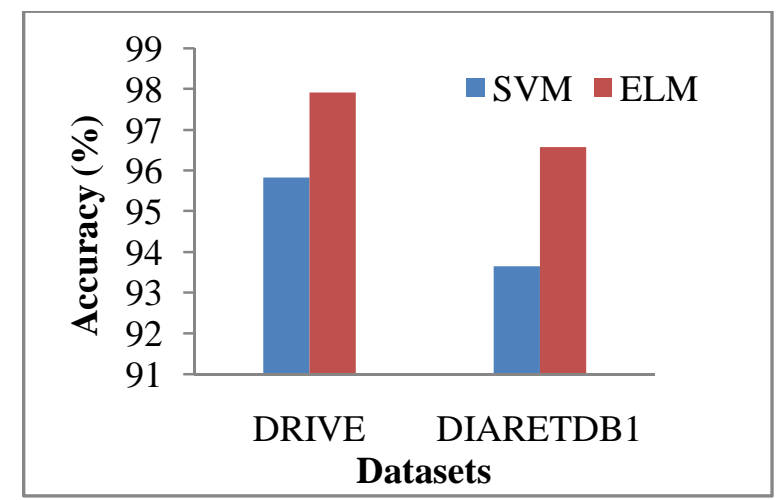

Chart-2: Comparison of Accuracy of Proposed Work for the Classifiers. 
The chart 2 shows the comparison graph of accuracy produced by the proposed work with the classifier SVM and ELM for DRIVE and DIARETDB1 dataset. This graph shows clearly that ELM produces maximum accuracy than SVM for the proposed technique.

\section{CONCLUSIONS}

Automatic detection of diabetic retinopathy turns out to be active research because of recent spread of these diseases largely. The first manifestation of diabetic retinopathy is microaneurysms which visible as a small reddish dot in human retinal image. The number of microaneurysms is the essential parameter used to identify the severity of the diabetic retinopathy. Detection of diabetic retinopathy at early stage can reduce the development of diseases significantly. This work is proposed to detect the diabetic retinopathy at early stage automatically which helps to cure fully or helps to reduce its growth. Here patients retinal images are captured intially and they are stored in a database. After that, they are preprocessed to reduce the noise and to enhance. Then candidate regions are extracted from the image along with blood vessels are removed to effectively extract the candidate regions. And MAs are enhanced by Gabor filter and from that different feature are extracted and they are given for the classification. The multi class classifier SVM and ELM taken as a classifier here confirms its efficient performance and proved for automatic detection of diabetic retinopathy in retinal images.

This work can be further extended in future with detecting Exudates, optic disk, vascular structures by employing hybrid techniques to produce best results in reduced complexity and time.

\section{REFERENCES}

[1] IDF Diabetes Atlas, 4th edition. International Diabetes Federation, 2009.

[2] A Ramachandran, AK Das, SR Joshi, CS Yajnik, S Shah, KM Prasanna Kumar, "Current Status of Diabetes in India and Need for Novel Therapeutic Agents". SUPPLEMENT TO JAPI • JUNE 2010 • VOL. 58.

[3] M. Usman Akram, Shehzad Khalid, Shoab A.Khan"Identification and classification of microaneurysms for early detection of diabetic retinopathy". 2012 Elsevier Ltd.

[4] Luca Giancardo, Fabrice Meriaudeaub, Thomas P. Karnowskia, Kenneth W., et al "Microaneurysms Detection with the Radon Cliff Operator in Retinal Fundus Images", Medical Imaging, 2010.

[5] S. Jiménez, P. Alemany, F. Nún ez Benjumea, C. Serrano, B. Achab, I. Fondón,F. Carral a, C. Sáncheza, "Automatic detection of microaneurysms in color fundus images",2012

[6] C.Sinthanayothin, J.F.Boyce, H.L.Cook, T.H.Williamson, Automated localisation of the optic disc, fovea and retinal blood vessels from digital colour fundus images, BritishJournal of Ophthalmology 83(1999)902-910.

[7] J.Sung, S.Y.Bang, S.Choi, A Bayesian network classifier and hierarchical Gabor features for handwritten numeral recognition, Pattern Recognition Letters 27(1) (2006)66-75.

[8] Lili Xu, Shuqian Luo," A novel method for blood vessel detection from retinal images", BioMedical Engineering OnLine 2010, 9:14.

[9] T. Chanwimaluang and G. Fan, "An Efficient Blood Vessel Detection Algorithm for Retinal Images using Local Entropy Thresholding", in Proc. of the 2003 IEEE International Symposium on Circuits and Systems, Bangkok, Thailand, May 25-28, 2003.

[10] N. R. Pal and S. K. Pal, .Entropic thresholding, Signal processing, vol. 16, pp. 97.108, 1989.

[11] La“y, B., Baudoin, C., and Klein, J.C., Automatic detection of microaneurysms in retinopathy fluoroangiogram, Proceedings of the SPIE, 432, 165, 1983.

[12] J.H. Hipwell, F. Strachan, J.A. Olson, K.C. McHardy, P.F. Sharp, and J.V. Forrester. Automated detection of microaneurysms in digital redfree photographs: a diabetic retinopathy screening tool. Diabetic Medicine, 17:588-594, 2000.

[13] G. Yang, L. Gagnon, S. Wang, and M.-C. Boucher. Algorithm for detecting micro-aneurysms in low resolution color retinal images. In Vision Interface 2001, pages 265-271, Ottawa, June 7-9 2001.

[14] A.D.Fleming,S.Philip,K.A.Goatman,J.A.Olson,P.F.Shar p,Automated microaneurysm detection using local contrast normalization and local vessel detection, IEEE Transactions on Medical Imaging 25(9)(2006)12231232.

[15] Retinopathy Online Challenge WebSite.The University of Iowa and the ROC Organizers (Online).Availablefrom:/http://roc.healthcare.uiowa.edu / results.phpS.

[16] M.Niemeijer,B.V.Ginneken,M.Cree,A.Mizutani,G.Quel lec,C.I.Sanchez,B.Zhang,R.Hornero,M.Lamard,C.Mura matsu,X.Wu,G.Cazuguel,J.You,A.M.Q.Li,Y.Hatanaka, B.Cochener,C.Roux,F.Karray,M.Garcia,H.Fujita,M.

Abramoff, Retinopathy online challenge: automatic detection of micro- aneurysms in digital color fundus photographs,IEEE Transactions on Medical Imaging 1(29)(2010)185-195.

[17] J. R. Quinlan. Induction of decision trees. Machine Learning, 1:81-106, 1986.

[18] Multi-SVM implementation by Cody Neuburger Florida Atlantic University, http://www.mathworks.in/matlabcentral/fileexchange/3 9352-multi-class-svm/content/multisvm.m

[19] DRIVE Database,: http:// www.isi.uu.nl/Research/Database/DRIVE

[20] DIARETDB1 Database, http://www2.it.lut.fi/project/imageret/diaretdb1/. 
[21] G.-B.Huang, Q.Y.Zhu, and C.K.Siew, Extreme learning machine: a new learning scheme of feed forward neural networks, in: Proceedings of the International Joint Conference on Neural Networks (IJCNN2004), Budapest, 2004, pp. 985-990.

[22] G.-B.Huang, Q.Y. Zhu, C.K.Siew, Extreme learning machine: theory and applications, Neuro computing 70 (2006) 489-501.

[23] Erkan Tanyildizi," Color texture image classification based on fractal features and extreme learning machine", Firat University, Technology Faculty, Department of Software Engineering, 23100, Elazig 